\title{
Investigating rainfall-induced unsaturated soil slope instability: a meshfree numerical approach
}

\author{
L. M. Dakssa \& I. S. H. Harahap \\ Civil Engineering Department, \\ Universiti Teknoloji PETRONAS, Malaysia
}

\begin{abstract}
It is a common phenomenon, especially, in tropical and subtropical regions that a standing soil slope fails during or immediately after heavy or prolonged rainfall events. A possible reason is that rainwater infiltration affects the pore-water pressure distribution in soil slope. While negative pore-water pressures add to the stability of soil slopes, positive pore-water pressures disrupt the existing stability. The changes in pore-water pressure in the soil are handled through infiltration/seepage analyses. Conventionally, numerical approach to seepage analysis is carried out by the mesh-based finite element software SEEP/W. More recent studies, however, indicate SEEP/W software yields appalling numerical oscillations near the wetting fronts as seepage progresses through unsaturated soils. In view of seeking an alternative approach, in this paper, a meshfree smoothed particle hydrodynamics (SPH) method was used to simulate infiltration into and seepage through unsaturated soil slope. The governing flow equation was developed by incorporating seepage force into the Navier-Stokes equation. Numerical examples were executed to test the capability of the SPH scheme in mimicking both infiltration and seepage. It was confirmed that the SPH method is versatile in that new physics of flow can be incorporated during program coding with ease. Besides, the simulation results indicate that SPH numerical approach can be considered as a better seepage analysis method as, unlike the mesh-based finite element, it does not suffer from mesh distortions when used for simulating large deformations - the case in landslides.

Keywords: continuum mechanics, hydraulic conductivity, infiltration, large deformation, matric suction, meshfree numerical methods, seepage, SPH.
\end{abstract}




\section{Introduction}

Analysis of infiltration and seepage through unsaturated soils plays a significant role in geotechnical hazards investigation. Geotechnical applications of seepage include, but not limited to, soil volume change predictions, contaminants transport through porous media, slope stability analysis, design of earth structures, such as earth dams, earth and rock fill dams, dykes, etc. Unsaturated soil slope instability (which is the target of the present paper), in the context of tropical and subtropical regions, occurs due to rainwater infiltration during heavy or prolonged rainfall events whereby the infiltrating rainwater disturbs the existing moisture equilibrium in the soil slope. The moisture disequilibrium is manifested mainly in terms of rise in the water content of the slope, thereby leading to reduction in the matric suction of the soil, which is thought to contribute to the stability of the soil slope. On the other hand, water content changes in unsaturated soil slopes due to rainwater infiltration lead to changes in the existing stresses. The change in the existing stresses in the soil will, in turn, cause the inevitable soil deformation and thereby soil slope instability.

Landslide predictions are usually made in terms of time and depth of failure so that residents residing nearby could get time to escape. To do so, knowledge of soil hydraulic conductivity and other relevant soil properties are required as fine-grained soils behave differently compared to the coarse-grained ones. As soil is naturally both heterogeneous and anisotropic, seepage analysis is usually carried out after making some sagacious assumptions. Therefore, in the current research, the soil under investigation is assumed to be heterogeneous and anisotropic, which, respectively, mean the soil engineering properties are both location and direction dependent.

Traditionally, seepage modelling is carried out, analytically, using Darcy's law or flownet method of Terzaghi if respective conditions are met and, if not, numerically, using finite difference (FD) or finite element (FE) methods. However, the existing numerical methods (FD and FE) have inherent difficulties for landslide modelling because of mesh-distortion in case of FE method and because of inefficient use of regular grids for irregular geometries in the case of FD method. In the current research, the objective is to induce rainwater infiltration and to simulate seepage in unsaturated soil slope using the smoothed particle hydrodynamics (SPH) - a meshless numerical technique.

\section{Methodology: smoothed particle hydrodynamics}

Smoothed particle hydrodynamics (SPH) is a macroscopic numerical approach initially developed for simulating astrophysical phenomena, in 1977 [1] and, later, its applications were reported in different areas of research, including free surface flows, flow thorough porous media, etc. SPH meshless numerical method formulation is based on interpolation theory, and two essential concepts dictate its formulation: (i) kernel approximation (ii) particle approximation. 


\subsection{Kernel approximation}

\subsubsection{Kernel approximation of field functions}

Kernel approximation of field functions is, in essence, the representation of the field function(s) in integral form. This is achieved by multiplying an arbitrary field functions with a smoothing kernel function. Therefore, a function $A$ of a three-dimensional position vector $\boldsymbol{x}$ (or an estimate of the function $A(\boldsymbol{x})$ at location $\boldsymbol{x}^{\prime}$ ) can be expressed in integral form:

$$
A(x)=\int_{\Omega} A\left(x^{\prime}\right) \delta\left(x-x^{\prime}\right) d x^{\prime}
$$

where, $\delta\left(x-x^{\prime}\right)$ is the Dirac delta function, given by:

$$
\delta\left(x-x^{\prime}\right)= \begin{cases}1 & \text { for } x=x^{\prime} \\ 0 & \text { elsewhere }\end{cases}
$$

where, $\Omega$ is the volume of the integral containing $x ; x^{\prime}$ is a new independent variable.

In the above expression, the function $A(x)$ is exact or rigorous, so long as the Dirac delta function is used and $A(x)$ is continuous in $\Omega$. In SPH, however, the Dirac delta function needs to be replaced by the smoothing (weighting) function $W\left(x-x^{\prime}, h\right)$ in which case it will become an approximate representation of $A(\boldsymbol{x})$. The SPH form of a function approximation (or kernel approximation) is, therefore:

$$
A(x)=\int_{\Omega} A\left(x^{\prime}\right) W\left(x-x^{\prime}, h\right) d x^{\prime}
$$

where, $W$ is called the kernel or smoothing function; $h$ is the smoothing length, and demarcates the influence area of the smoothing function. It needs to be noted, however, that eqn (3) gives an approximate representation of the integral of a field function as long as $W$ is not a Dirac delta function; and, hence, the name kernel approximation.

\subsubsection{Kernel approximation of derivative of a function}

As equations of computational fluid dynamics problems are mostly PDEs of second degree [1], an appropriate approximation of the function derivatives is of profound importance. Accordingly, the kernel approximation of the divergence of the field function $A(\boldsymbol{x})$ (for vector quantity) is, therefore:

$$
\nabla \cdot A(x)=\int_{\Omega} \nabla \cdot A\left(x^{\prime}\right) W\left(x-x^{\prime}, h\right) d x^{\prime}
$$

After applying the divergence theorem, it is always the case that the divergence operation on the primed coordinate in eqn (4) is transferred to the gradient of the smoothing function in SPH numerical approach, which entails rewriting eqn (4) as:

$$
\nabla \cdot A(x)=-\int_{\Omega} A\left(x^{\prime}\right) \cdot \nabla W\left(x-x^{\prime}, h\right) d x^{\prime}
$$


Note that dot product is used in eqn (5). Similarly, the gradient of the function (for scalar quantity) is expressed as:

$$
\nabla A(x)=-\int_{\Omega} A\left(x^{\prime}\right) \nabla W\left(x-x^{\prime}, h\right) d x^{\prime}
$$

It can be said, therefore, that the spatial derivative of a field function can be evaluated from the values of the field function and the spatial derivative of the smoothing function. It should also be noted that the negative sign outside the integral sign in eqns (5) and (6) can be removed if the spatial derivative of the kernel function is taken with respect to $\mathbf{x}$ instead of the primed $\mathbf{x}$ (i.e., $x^{\prime}$ ).

\subsection{Particle approximation}

\subsubsection{Particle approximation of field function}

Particle approximation is another key operation in SPH numerical formulation; and is the means of transforming the continuous kernel approximation (in integral form) into the summation over all particles at the discrete points in the support domain. Particles carry mass, $m$, velocity, $v$, and other quantities specific to the given problem; and, are regarded as interpolation points, analogous to the grid nodes in mesh-based numerical methods. Therefore, equations that govern the evolution of fluid quantities are expressed as summation interpolants with the help of smoothing function. Eqn (9) can, then, be approximated in a summation form as:

$$
A(x) \approx \sum_{j=1}^{N} A\left(x_{j}\right) W\left(x-x_{j}, h\right) \frac{m_{j}}{\rho_{j}}
$$

where $N$ is the total number of particles in the support domain; $m_{j}$ and $\rho_{j}$ are the mass and density of particle $j$, respectively. And also, it should be noted that the infinitesimal volume $\mathrm{d} \mathbf{x}^{\prime}$ is replaced by the finite volume $\Delta V=m_{j} / \rho_{j}$.

From eqn (7), it is possible to infer that the approximate value of a function at any discrete point can be obtained using the weighted average of those values of the function at all other particles in the influence domain of that particle.

Following similar argument, the particle approximation for a function at particle (point) $i$ may be written as in eqn $(8)[1,2]$ :

$$
A\left(x_{i}\right)=\sum_{j=1}^{N} \frac{m_{j}}{\rho_{j}} A\left(x_{j}\right) W\left(x_{i}-x_{j}, h\right)
$$

\subsubsection{Particle approximation of gradient and divergence of field functions}

From eqn (8), it can be said that the continuous integral representation of the field function can be expressed in a discretized summation form, which is one of the favourable qualities of the SPH method as that renders the use of background mesh for numerical integration unnecessary [1]. Such conversion of the governing mathematical equations to a workable SPH numerical scheme, however, requires some fundamental techniques as discussed below. 
Transformation of the PDEs to the SPH discretized summation form, for instance, can be achieved by different ways. One way is with the help of integration by parts and Taylor series expansion. Suppose $A$ is a scalar field function representing any physical variable and is defined in a given domain of interest. Its gradient can be formulated in a similar manner to eqn (8) as;

$$
(\nabla A)_{i}=\sum_{j=1}^{N} \frac{m_{j}}{\rho_{j}} A_{j} \nabla_{i} W_{i j}
$$

where,

$$
\begin{array}{r}
\nabla_{i} W_{i j}=\frac{x_{i}-x_{j}}{\left|x_{i}-x_{j}\right|} \frac{\partial W_{i j}}{\partial r_{i j}}=\frac{x_{i j}}{r_{i j}} \frac{\partial W_{i j}}{\partial r_{i j}}=\nabla_{x i} W_{i j}=-\nabla_{x^{\prime} i} W_{j i} \\
W_{i j}=W\left(x_{i}-x_{j}, h\right)
\end{array}
$$

Applying some basics of vector calculus, other forms of the gradient equations can also be formulated. For instance, putting $\rho$ inside the gradient operator and applying the chain rule, eqn $(9 \mathrm{c})$ can be obtained. The introduction of mass and density into SPH particle approximation is to facilitate numerical approaches in hydrodynamic problems, where density is a key parameter.

$$
\nabla(\rho A)=\rho \nabla A+A \nabla \rho \Rightarrow \nabla A=\frac{1}{\rho}[\nabla(\rho A)-A \nabla \rho]
$$

And, re-writing in SPH particle approximation form;

$$
(\nabla A)_{i}=\frac{1}{\rho_{i}}\left(\sum_{j=1}^{N} m_{j}\left(A_{j}-A_{i}\right) \nabla_{i} W_{i j}\right)
$$

The third possible way of approximating the gradient of the field variable $A$ is incorporating the SPH kernel and particle approximation on a gradient of the unity.

$$
\nabla 1=\int 1 \cdot \nabla W\left(x-x^{\prime}, h\right) d x^{\prime}=\sum_{j=1}^{N} \frac{m_{j}}{\rho_{j}} \nabla_{i} W_{i j}
$$

And, obviously, the gradient of the unity is zero. Therefore, eqn $(9 \mathrm{e})$ can be re-written in the form containing both particles $i$ and $j$ as:

$$
\nabla 1=\rho_{i} A_{i}\left(\sum_{j=1}^{N} \frac{m_{j}}{\rho_{j}} \nabla_{i} W_{i j}\right)=0
$$

Adding eqns (9a) and (9f) give another discretized (or discrete) form of the gradient of a field variable and is presented in eqn $(9 \mathrm{~g})$.

$$
(\nabla A)_{i}=\rho_{i} \sum_{j=1}^{N} \frac{m_{j}}{\rho_{j}}\left(A_{i}-A_{j}\right) \nabla_{i} W_{i j}
$$

As it can be seen in eqn $(9 \mathrm{~g})$, unlike in eqn (9a), the field variable difference is introduced into the discrete particle approximation. Eqn $(9 \mathrm{~g})$ is preferred to eqn (9a), as, in such asymmetric forms, the presence of the variable difference can reduce errors arising from particle inconsistency problems [1]. As a fourth 
approach, again inserting $\left(\frac{1}{\rho}\right)$ in the gradient operator and applying the chain rule;

$$
\nabla\left(\frac{A}{\rho}\right)=\frac{1}{\rho} \nabla A-\frac{1}{\rho^{2}} A \nabla \rho \Rightarrow \nabla A=\rho\left[\nabla\left(\frac{A}{\rho}\right)+\frac{1}{\rho^{2}} A \nabla \rho\right]
$$

And, the SPH equivalent of eqn $(9 \mathrm{~h})$ is;

$$
(\nabla A)_{i}=\rho_{i}\left(\sum_{j=1}^{N} m_{j}\left(\frac{A_{j}}{\rho_{j}^{2}}+\frac{A_{i}}{\rho_{i}^{2}}\right) \nabla_{i} W_{i j}\right)
$$

Lastly, if one wishes to develop SPH particle approximation for $\left(\frac{1}{\rho} \nabla A\right)$;

$$
\left(\frac{1}{\rho} \nabla A\right)_{i}=\frac{1}{\rho_{i}}\left(\sum_{j=1}^{N} \frac{m_{j}}{\rho_{j}} A_{j} \nabla_{i} W_{i j}\right)
$$

In order to obtain a symmetric equation, the concept of a gradient on the unity can be applied here too.

$$
\nabla 1=\frac{A_{i}}{\rho_{i}}\left(\sum_{j=1}^{N} \frac{m_{j}}{\rho_{j}} \nabla_{i} W_{i j}=0\right)
$$

Adding eqns (9j) and (9k) to obtain a symmetric equation;

$$
\left(\frac{1}{\rho} \nabla A\right)_{i}=\sum_{j=1}^{N} m_{j}\left(\frac{A_{i}+A_{j}}{\rho_{i} \rho_{j}}\right) \nabla_{i} W_{i j}
$$

And, from eqn (9h);

$$
\frac{1}{\rho} \nabla A=\nabla\left(\frac{A}{\rho}\right)+\frac{1}{\rho 2} A \nabla \rho \Rightarrow\left(\frac{1}{\rho} \nabla A\right)_{i}=\sum_{j=1}^{N} m_{j}\left(\frac{A_{j}}{\rho_{j}{ }^{2}}+\frac{A_{i}}{\rho_{i}{ }^{2}}\right) \nabla_{i} W_{i j}
$$

Therefore, eqns (9a), (9d), (9g), (9i), (9j), and (9l) are all correct particle approximations of any scalar field variable $A$ though the symmetric equations are thought to yield accurate results [1]. Following similar procedures as in the case of the gradient of a field variable, the divergence of the field variable can also be expressed in a particle approximation scheme by simply replacing the gradient with the divergence operator.

Note that the negative sign in eqn (5) has been dropped in the above equations, because, here, the spatial derivative of the smoothing function $\nabla W$ is taken with respect to particle $i$, and not with respect to particle $j$.

\subsection{Smoothing functions}

Smoothing (also, called weighting) function is at the core of the SPH formulation. Spatial discretization of field variables is based on a set of points (particles, in SPH nomenclature), instead of grid nodes, which are commonly used in mesh-based numerical methods, such as FD and FE methods. It is, thus, 
with the use of kernel interpolation that field variables, such as velocity, pressure, density, stress, etc., are approximated at any point (i.e., at any discrete point) in the support domain. Accordingly, there are several kernel functions being used in SPH numerical method. The piecewise cubic-spline function, commonly known as $B$-spline, suggested by Monaghan and Lattanzio, quoted in [1], is popular among SPH numerical modelers, such as SPHyscis code developers [3]. The same function is used here.

$$
W(r, h)=\alpha_{d} \begin{cases}\frac{1-\frac{3}{2} q^{2}+\frac{3}{4} q^{3}}{\frac{(2-q)}{4}^{3}} & ; 1 \leq q \leq 2 \leq 1 \\ 0 & ; q \geq 2\end{cases}
$$

where,

$$
q=\frac{\left|x_{i}-x_{j}\right|}{h}=\frac{r}{h} \text { is the relative distance between the two points, } x_{i} \text { and } x_{j},
$$
$\left|x_{i}-x_{j}\right|=r$ is the distance between the two particles. And, $\alpha_{d}$ is given by $\frac{10}{7 \pi h^{2}}$ and $\frac{1}{\pi h^{3}}$ for two and three dimensions, respectively.

The glaring shortcoming of spline functions is that their second derivative is a piecewise linear function, and, therefore, the stability properties can be inferior to those of smoother kernels [1]. This could, probably, be one of the reasons why the spatial first derivative of the cubic spline smoothing functions is widely used in the emerging literature.

\section{Applications: seepage modelling and simulation}

\subsection{Governing equation}

The numerical software program SEEP/W is being used for modelling seepage through both saturated and unsaturated soils [4]. SEEP/W was developed using the $h$-based form of the Richards' equation (RE) [5]. However, its reliability for numerical simulation of seepage through unsaturated soil slopes has become questionable. As noted in the works of Phoon et al. [5], Karthikeyan et al. undertook a thorough study of both one- and two-dimensional seepage through a porous media using SEEP/W, and came up with the conclusion that numerical oscillation popped up near the wetting front when water started to flow via unsaturated soils. The same study revealed also that, due to the numerical oscillations, unphysical results, such as drying of the soil close to the influx boundary, were obtained. Also, the Navier-Stokes (NS) equation has been widely 
used to model flow through porous media by several researchers, for instance, Narsilio et al. [6], Jiang and Sousa [7], Morris et al. [8], and Pereira et al. [9]. The Navier-Stokes equation models flow at the micro or pore-scale level. Shao [10] investigated the interaction between water waves and porous medium using what he dubbed Navier-Stokes-type equation. NS equation was also used by Monaghan [11] for simulating free surface flow using SPH, with the exception that artificial viscosity term was used instead of the laminar viscosity. In a separate study Morris et al. [12] employed the same continuity and momentum equations to model incompressible low Reynolds number flows.

A similar formulation for the porous media could be carried out with the exception that the $f$ values in NS equations may be accounted for a bit differently, as there will be a frictional force created when water flows in porous media. Following the work of Shao [10], the momentum equation for the flow through soils can be given as in eqns (11) and (12).

$$
\begin{gathered}
\frac{1}{\rho} \frac{D \rho}{D t}+\nabla \cdot \boldsymbol{V}_{s}=0 \\
\frac{D \boldsymbol{V}_{s}}{D t}=-\frac{1}{\rho} \nabla P+v \nabla^{2} \boldsymbol{V}_{s}+\boldsymbol{g}-\eta \gamma_{w} \nabla\left(\frac{\boldsymbol{V}_{s}}{\boldsymbol{K}}\right)
\end{gathered}
$$

where, $\boldsymbol{V}_{s}$ is seepage (also known as actual, interstitial) velocity vector, $\eta$ is the porosity of the soil, $\boldsymbol{K}$ is the hydraulic conductivity tensor for the soil, $\gamma_{w}$ is the unit weight of water, $\rho$ is water density, $\boldsymbol{g}$ is gravity, $P$ is pressure.

The last term in eqn (12) refers to the seepage force and is included in the momentum equation as an external drag force, as it acts to hinder water flow in the soil. Here, Darcy's macroscopic law is employed. It is to be noted that Darcy's velocity is soil porosity multiplied by the seepage velocity. The hydraulic conductivity tensor in the last term is expressed as a function of the pore-water pressure for unsaturated soils. In the current study, the Gardner's equation [4] is used to express the $\mathbf{K}$ as a function of matric suction.

$$
\boldsymbol{K}=\frac{\boldsymbol{K}_{s}}{1+a\left[\frac{\left(u_{a}-u_{w}\right)}{\gamma_{w}}\right]^{n}}=\frac{\boldsymbol{K}_{s}}{1+a\left(\frac{P}{\gamma_{w}}\right)^{n}}
$$

where, $\mathbf{K}$ and $\boldsymbol{K}_{s}$ are the hydraulic conductivity coefficients for unsaturated and saturated soils, respectively; $\left(u_{a}-u_{w}\right)$, which is equal to the $P$ value in eqn (12), is the matric suction; and $a$ and $n$ are constants. Values of $\mathrm{a}=0.1$ and $\mathrm{n}=2$ were used (see [4]) and hydraulic conductivity in the z-direction was assumed to be half of the hydraulic conductivity in the z-direction in the current research. And also, it is worth noting that SPH was originally invented for modelling flows of compressible fluids, and, thus its application to incompressible fluid flows needs some treatment to ensure density variation within a certain limit is maintained. In order to circumvent the difficulty of solving the pressure term for incompressible fluids, previous research works have resorted to using equation of state (EoS), as discussed in the next section. 


\subsection{Equation of state}

For the standard SPH for compressible fluids, particle motion is triggered by pressure gradient, which is normally calculated using equation of state (EoS). However, for the case of incompressible fluids, applying and solving the pressure using an incompressible fluid EoS dictates the adoption of small timestep [1]. This constraint has led to the adoption of artificial compressibility for solving the pressure gradient in the governing equation, the approach which is dubbed quasi-incompressibility by some researchers. Accordingly, Monaghan [11] modified the EoS suggested for water by Batchelor (also, cited in [11]), for describing sound waves and used it for simulating free surface flows, and the same equation has been frequently used by several emerging literatures. In this research too the same EoS is used as given in eqn (15). Moreover, Bui et al. [13] applied the same EoS in their formulation of SPH for soil mechanics with some successes.

$$
P=B\left[\left(\frac{\rho}{\rho_{o}}\right)^{\gamma}-1\right]
$$

where, $\gamma$ is constant and is taken to be 7 for most circumstances, $\rho_{o}$ is the reference density, $B$ is a problem dependent parameter for limiting the maximum density gradient and, in most cases, can be taken as the initial pressure $[1,11]$.

\subsection{Boundary treatment}

Boundary treatment entails special consideration in SPH, as particle deficiency near or on the boundary impairs full exploitation of the scheme. Monaghan [11], also reported in [1], suggested the use of ghost particles near or on the boundaries so that high repulsive force is created to prevent fluid particles from unphysically penetrating a solid boundary. Such penalty force approach to prevent interior fluid particles from penetrating the boundary is based on the Lennard-Jones molecular force approach. Another approach, in which the Hertzian contact theory was used, was also developed by Bui et al. [13]. For the current research we intend to use the Monaghan approach as given in eqn (15).

$$
P B_{i j}= \begin{cases}D\left[\left(\frac{r_{o}}{r_{i j}}\right)^{a}-\left(\frac{r_{o}}{r_{i j}}\right)^{b}\right] \frac{x_{i j}}{r_{i j}{ }^{2}} & \left(\frac{r_{o}}{r_{i j}}\right) \leq 1 \\ 0 & \text { elsewhere }\end{cases}
$$

where, as in [1], $a$ and $b$ are taken to be 12 and 4, respectively, although Monaghan [11] proposed 4 and 2, respectively, with the conditions that $a>b$, always. He also suggested that $a$ and $b$ could also be taken as 12 and 6 , respectively, without significant changes in the results. $D$ is a problem dependent parameter and is usually taken to be the square of the largest velocity [1], and $r_{o}$ is selected to be approximately equal to the initial particle spacing. 


\subsection{Time integration}

Here, the predictor-corrector method is used. The predictor step predicts a new value, and the corrector step improves the accuracy of that value. The predictor step is undertaken only once for each of the iterations, while the corrector step is continued until the required level of accuracy is reached. There are several predictor-corrector methods though for the current paper we stick to the Euler predictor-corrector method (some prefer to name it modified Euler method). The basic idea behind the $2^{\text {nd }}$ order Euler predictor-corrector method is that the solution for a new timestep is predicted using the explicit Euler method and the final solution is corrected by applying the trapezoid rule as follows.

$$
\begin{gathered}
\phi^{(n+1)^{*}}=\phi^{n}+f\left(t_{n}, \phi^{n}\right) \Delta t \\
\phi^{(n+1)}=\phi^{n}+\frac{1}{2}\left[f\left(t_{n}, \phi^{n}\right)+f\left(t_{n+1}, \phi^{(n+1)^{*}}\right)\right]
\end{gathered}
$$

For the sake of stability, the timestep, $\Delta \mathrm{t}$, needs to be checked against several stability requirements. Detailed reading regarding these stability conditions can be made in $[1,11]$.

\section{Numerical examples and discussions}

In this section numerical calculations and discussions will be made.

Data used:

Saturated hydraulic conductivity: $10^{-4} \mathrm{~m} / \mathrm{s}$

Rainfall intensity (surface-flux) $: 10^{-4} \mathrm{~m} / \mathrm{s}$

Porosity : 0.6

Slope angle $\quad:-40^{\circ}$

Kinematic viscosity $: 1 \times 10^{-6} \mathrm{~m}^{2} / \mathrm{s}$.

Figure 1 shows the configuration of the slope physical model before and after the onset of infiltration. Figure 1 (a) depicts the initial slope configuration, the white colour representing the soil particles and the blue water particles. For the current simulation one main program with a number of subroutines were coded to develop the initial configuration. A separate set of program codes were also coded for inducing infiltration and running the seepage simulation. Different simulation examples were carried out for different timesteps and simulation times. The left and the bottom boundaries were treated as no-flow boundaries, the other sides were considered porous. In the coding, it was possible to record automatically, in a separate file, the positions of each particle, their respective velocities, densities and pore-water pressures. Those data files were used for the graphics using MATLAB. As can be noted from Figure 1 (b)-(d), the SPH scheme is a suitable means for capturing different aspects of infiltration into and seepage through unsaturated soils. 


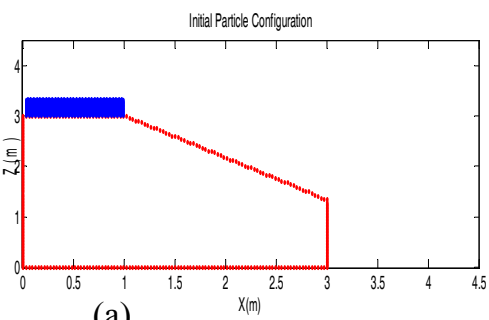

(a)

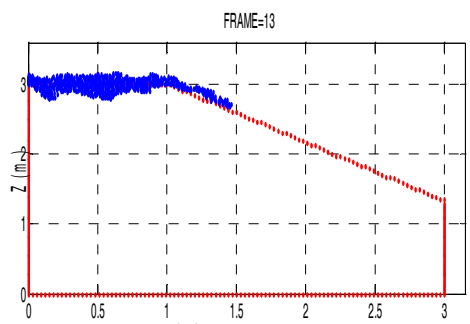

(c) $x(m)$

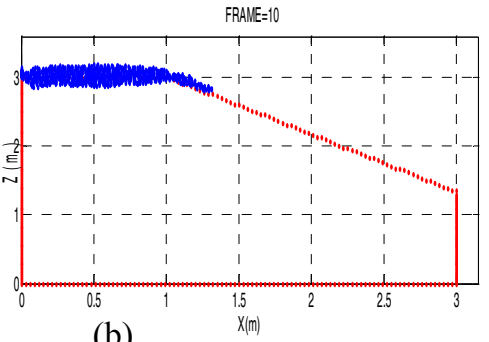

(b)

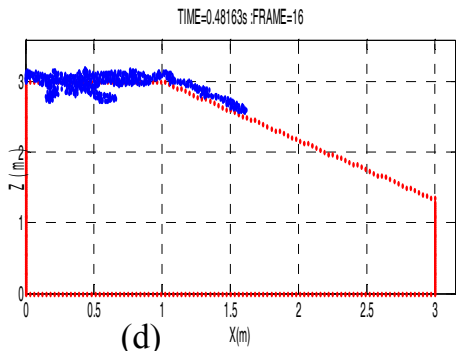

(d)

Figure 1: Example of SPH simulation for simulation time of approximately $0.5 \mathrm{sec}$ and a timestep of $0.02 \mathrm{sec}$.

\section{Conclusions}

In this paper, the SPH scheme was employed to simulate infiltration into and seepage through unsaturated soil slope. Only single-phase flow conditions were considered in the current study based on the evidences available in the emerging literature regarding the applicability of such cases for most engineering applications. Due to numerical instability, the soil slope receiving surface flux (i.e., infiltration) was limited to the crest of the slope, and the left, bottom and the inclined portions of the slope were considered regions of zero-surface flux. The governing flow equations for the current study were formulated by incorporating the frictional drag force during seepage through a porous media into the fundamental Navier-Stokes flow equations. Several numerical test problems were executed to check the capability of the new approach, yielding meaningful results, in our view. The simulation output for the current study included respective particle velocities, positions of each particle (which indicate depth of infiltration), pore-water pressures, and densities. Pore-water pressure data are indicative of slope instability/stability as reduction of the matric suction leads to loss of soil's shear strength and hence slope instability. The experimental work to validate the numerical results is in progress. Acknowledging the need for further improvements, especially, with respect to numerical stability problems, it is fair to say that the current work has shed light regarding the potential capability of the SPH numerical model to simulate conditions leading to unsaturated soil slopes. 


\section{References}

[1] Liu, G.R. and Liu, M.B., Smoothed particle hydrodynamics - a meshfree particle method. World Scientific Publishing, Singapore, 2003.

[2] Bui, Ha H., Sako, K. and Fukagawa, R., Numerical simulation of soil-water interaction using smoothed particle hydrodynamics (SPH) method. Journal of Terramechanics 44, 2007, 339-346.

[3] Gesteira, M.G, Rogers, B.D, Dalrymple, R.A, Crespo, A.J.C. and Narayanaswamy, M., User Guide for the SPHysics Code v2.0, Online http://wiki.manchester.ac.uk/sphysics, 2010.

[4] Ferdlund, D.G. and Rahardjo, H., Soil mechanics for unsaturated soils. John Wiley and Sons, New York, 1993.

[5] Phoon, K.K., Tan, T.S. and Chong, P.C., Numerical simulation of Richards' equation in partially saturated porous media: under-relaxation and mass balance. Geotech Geol Eng 25:525-541, 2007.

[6] Narsilio, G.A., Buzzi, O, Fityus, S., Yun, T.S. and Smith, D.W., Upscaling of Navier-Stokes equations in porous media: theoretical, numerical and experimental approach. Computers and Geotechnics 36, 1200-1206, 2009.

[7] Jiang, F. and Sousa, A.C.M., Smoothed particle hydrodynamics modelling of transverse flow in randomly aligned fibrous porous media. Transp porous med 75, 17-33, 2008.

[8] Morris, J.P., Zhu, Y. and Fox, P.J., Parallel simulations of pore-scale flow through porous media Computers and Geotechnics 25, 227-246, 1999.

[9] Pereira, G.G., Prakash, M. and Cleary, P.W., SPH modelling of fluid at the grain level in a porous medium. Applied Mathematical Modelling 35, $1666-1675,2011$.

[10] Shao, S., Incompressible SPH flow model for wave interactions with porous media. Coastal Engineering, 57, 304-316, 2010.

[11] Monaghan, J.J., Simulating free surface flows with SPH. Journal of Computational Physics, 110, 399-406, 1994.

[12] Morris, J.P., Fox, P.J. and Zhu, Y., Modelling low Reynolds number incompressible flows using SPH, Journal of Computational Physics 136, 214-226, 1997.

[13] Bui, Ha H., Fukagawa, R. and Sako, K., Smoothed particle hydrodynamics for soil mechanics. Numerical Methods in Geotechnical Engineering (eds.), Taylor and Francis, 2006. 